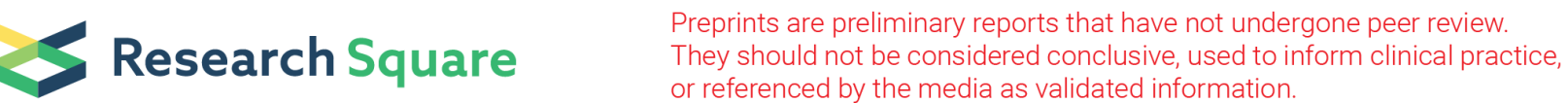

\section{A Home-Based, Post-Discharge Early Intervention Program Promotes Motor Development and Physical Growth in the Early Preterm Infants: A Prospective, Randomized Controlled Trial}

\section{Juan Fan}

Chongqing Medical University Pediatric College: Chongqing Medical University Affiliated Children's Hospital

\section{Jianhui Wang}

Chongqing Medical University Pediatric College: Chongqing Medical University Affiliated Children's Hospital

\section{Xianhong Zhang}

Chongqing Medical University Pediatric College: Chongqing Medical University Affiliated Children's Hospital

\section{Ruiyun He}

Chongqing Medical University Pediatric College: Chongqing Medical University Affiliated Children's Hospital

\section{Shasha He}

Chongqing Medical University Pediatric College: Chongqing Medical University Affiliated Children's Hospital

\section{Mei Yang}

Chongqing Medical University Pediatric College: Chongqing Medical University Affiliated Children's Hospital

\section{Yujie Shen}

Chongqing Medical University Pediatric College: Chongqing Medical University Affiliated Children's Hospital

\section{Xiaojun Tao}

Chongqing Medical University Pediatric College: Chongqing Medical University Affiliated Children's Hospital

\section{Mei Zhou}

Chongqing Medical University Pediatric College: Chongqing Medical University Affiliated Children's Hospital

\section{Xiong Gao}

Chongqing Medical University Pediatric College: Chongqing Medical University Affiliated Children's Hospital 


\section{Lijun Hu ( $\sim$ hlj1116@163.com )}

Children's Hospital of Chongqing Medical University

\section{Research article}

Keywords: early preterm infants, early intervention, neurodevelopment, physical growth

Posted Date: October 7th, 2020

DOI: https://doi.org/10.21203/rs.3.rs-73613/v1

License: (c) (1) This work is licensed under a Creative Commons Attribution 4.0 International License. Read Full License

Version of Record: A version of this preprint was published at BMC Pediatrics on April 7th, 2021. See the published version at https://doi.org/10.1186/s12887-021-02627-x. 


\section{Abstract}

Background: Early intervention (EI) has been believed to improve the neurodevelopment of early preterm infants. However, its implementation in medical settings is time-consuming and resource-intensive, which limits its extensive use. In 2018, the Chinese Eugenics Association developed a home-based, postdischarge El program. This study aims at evaluating the effect of this El program on neurodevelopment and physical growth of early preterm infants through a prospective, partially blinded, randomized controlled trial, and a subsequent open phase.

Methods: A total of 73 infants born at $28^{+0} \llbracket 31^{+6}$ weeks' gestation with postmenstrual age of $36^{+0} \square$ $39^{+6}$ weeks were randomly allocated to the first early intervention, then standard care (EI-SC) group (37 infants), and the first standard care, then early intervention (SC-EI) group (36infants). Another 33 infants were retrospectively recruited as the reference group. The test of infant motor performance (TIMP) was the primary outcome, while development quotient (DQ) and anthropometric measures, including weight, length, and head circumference, were the secondary outcome. The assessment was carried out at the baseline (T0), termination of the randomized controlled trial (T1), and termination of the open phase (T2), respectively.

Results: During the randomized controlled trial, the EI-SC group had significantly higher delta change (T1T0) than the SC-El group in TIMP and anthropometric measures $(p<0.05)$, while no difference in DQ was noted between groups $(p>0.05)$. In terms of the delta change from T2 to T0, there was no significant difference in all outcomes between the EI-SC group and SC-El group ( $p>0.05)$. At the endpoint of T2, the EI-SC and SC-El group had similar TIMP and anthropometric measures, but much higher than the reference group.

Conclusions: These findings demonstrated that a home-based, post-discharge El program in this study was a practical approach to promote motor development and physical growth in early preterm infants.

Trial Registration: CHICTR, CTR1900028330, registered December 19, 2019, https:// http://www.chictr.org.cn/showproj.aspx?proj=45706

\section{Background}

In the last two decades, the survival of early preterm infants (EPI) has dramatically improved due to the tremendous progress of neonatal intensive care. However, these survivors' high incidence of neurodevelopmental impairment is still of great concern to the neonatologist and pediatricians(1). Studies reported that $53 \% \otimes 72 \%$ of EPI experienced learning difficulties and had weaker performance in academics than their term peers, and $15 \%$ of them would develop cerebral palsy $(2,3)$. Currently, to improve the long-term outcome of EPI, particularly the neurodevelopment, is a significant challenge in the pediatric field. 
Early intervention (EI) is believed to improve the neurological prognosis of infants born prematurely, who was at high risk for neurodevelopmental impairment(3-6). Preterm infants have considerable brain plasticity during the early stage of life, and their nervous system has excellent development potential(7). Applying El to preterm infants can provide benign stimulus to the nervous system, modify the immature brain tissue, shape the brain structures, and ultimately improve the infant neurodevelopment $(4-6,8)$. A large body of literature has also demonstrated the effect of El on promoting the physical growth of infants, including weight, length, and head circumference $(9,10)$.

Currently, most El program was carried out in the hospital, follow-up clinics, or community-based medical settings. Implementation of an El program is laborious, time-consuming, and resource-intensive, which limits its use on a large scale, particularly in some medical resource-limited regions. In 2018, the Chinese Eugenics Association developed a home-based, post-discharge El program and tried to extend it to all appropriate medical facilities in China, aiming to promote the global development of preterm infants. This program makes parents the front-line executors of El under the orientation of medical staff, and it seems to satisfy all requirements for an ideal El model: flexibility, low-cost, and active involvement of infants and their families(11). This program provides an innovative approach to carry out El. To our knowledge, so far, no literature regarding the effectiveness of this El program has been published internationally. This study was aimed to evaluate the impact of this home-based, post-discharge El program on neurodevelopment and physical growth in EPI.

\section{Methods}

\section{Study design}

This study was a single-center, prospective, partially blinded, randomized controlled trial (RCT), which was followed by an open phase. It was approved by the Institutional Review Board of Children's Hospital of Chongqing Medical University (No.2019-216) and was registered in ChiCTR (ChiCTR1900028330) with full protocol being available online. Written consent was obtained from the parents. Our study adhered to the CONSORT guidelines.

All enrolled infants were randomly allocated following simple randomization procedures (computerized random number) to the first early intervention, then standard care (EI-SC) group, or first standard care, then early intervention (SC-EI) group. The twins were allocated to the same group in favor of parental care. During the RCT period, infants in the El-SC group had the opportunity to have a 30-day El exposure superimposed with the standard care, while those in the SC-El group were only given standard care at this stage. The assessment was performed for all infants at baseline (T0) and 60 days later (T1, primary endpoint). In the following open phase, the infants initially allocated to the EI-SC group continued standard care, while those initially allocated to the SC-El group received a 30-day El due to the ethical consideration. A repeated assessment was scheduled at 120 days of study (T2, second endpoint). All assessment was blinded to the assessors. 
A sample of preterm infants meeting the same inclusion criteria was respectively recruited to provide a reference group in this study. The clinical data of these infants were extracted from our hospital follow-up dataset.

\section{Participants}

All EPI admitted to the Children's Hospital of Chongqing Medical University between Dec 1, 2019, and June 31, 2020, were eligible for this study. The inclusion criteria were as follows: (i) born at $28^{+0} \llbracket 31^{+6}$ weeks' gestation, with postmenstrual age of $36^{+0} \llbracket 39^{+6}$ weeks; (ii) stable vital signs, no oxygen requirement, reasonable daily weight gain; (iii) either of the parents being able to take round-the-clock care for the infants at home.

The exclusion criterion was as follows: (i) small for gestational age; (ii) presumed brain injury, including intraventricular hemorrhage $\geq$ grade II, various degree of periventricular leukomalacia, neonatal seizure; (iii) congenital or acquired sensory deficits; (iv) presence of a major dysmorphic feature, or laboratoryconfirmed chromosomal abnormality; (v) single-family, or parents with language barriers, neurodevelopment impairment, or other disabilities expected to interfere with the implementation of El; (vi) infants were given various interventions from specialist simultaneously, such as an occupational therapist, physiotherapist, and speech therapist; (vii) parents declined to participate.

\section{Implementation of the El program}

Once the parents signed the consent to participate in this study, a training session would be arranged soon. A didactic lecture was delivered to the parents to provide a holistic view of this El program, which was followed by a simulation workshop. This El program mainly consists of three sections: intellectual, physical, and social section, which could be implemented separately. The intellectual section includes a hearing-induced training and a vision-induced training; the physical section involved a whole-body massage; the social section includes the kangaroo care and a hearing-vision integrated training (the video was available with hyperlinks in additional file 1). A researcher with rehabilitation backgrounds conducted all workshops. The parents were only eligible for performing El independently after obtaining permission from the researcher.

Once the enrolled infant was discharged home, the parents should start the daily performance of EI promptly. Researchers would further assess the correctness of performance and suitability of the surrounding environment based on the video obtained from the parents. Parents should also fill out an information card daily, recording the time and duration of El, the operator, and infant response. A researcher was designated to collect such information.

\section{Standard care and follow-up clinic}

Standard post-discharge care was provided to all eligible infants, including a bimonthly follow-up clinic and scheduled immunization. Pertinent advice and booklets dedicated to home-care were delivered to all 
parents. The researchers recorded sporadic clinic visits due to infant unwellness.

Each follow-up clinic visit should perform the basic anthropometric measures, including weight, length, and head circumference $(\mathrm{HC})$. All EPI received a comprehensive neurological assessment due to their high risk for neurodevelopmental impairment. The follow-up information was stored in a hospital followup dataset.

\section{Outcome measures}

\section{Primary outcome measures}

Test of Infant Motor Performance (TIMP) during the RCT was the primary outcome in this study, which was widely used to evaluate the effect of an intervention on infant motor performance(12). TIMP consists of 42 items organized into two subsets (observed and elicit items). A total raw score is summed from individual item score, which was analyzed in this study.

\section{Secondary outcome measures}

Development quotient (DQ) is a ratio statistic reflecting the global development of infants related to criteria logged in authentic social contexts. Gross motor, fine motor, social development, adaptiveness, and language skills were the five subdomains assessed in this study. An overall general DQ score was calculated by summing these subdomains, and more than 85 is considered normal(13).

The anthropometric measures, including weight, length, and $\mathrm{HC}$ were also recorded and analyzed as the secondary outcomes in this study.

\section{Statistics}

The sample size for RCT was calculated to detect a difference of 0.5SD in the raw score of TIMP between groups (on a 2 -side test with $a=0.05 ; \beta=0.80$ ). A total of 28 infants were needed in each group. Assuming a follow-up rate of $85 \%$, the sample size was 33 in each group.

Clinical data were collected using Epidata 3.1 software, and all data analyses were carried out using SPSS 19.0. Normal distributed continuous data were expressed as the mean \pm SD, which was compared using one-way ANOVA between groups. The categorical data were expressed as a percentage, which was compared using the chi-square or Fisher's exact test between groups. A p value $<0.05$ was considered significant.

We calculated the delta change of TIMP, DQ, and anthropometric measures from T0 to T1, and compare the delta changes between the EI-SC group and SC-El group to verify the effect of El program versus standard care on infant development and physical growth. Furthermore, the delta changes from T0 to T2 were also calculated and compared to test whether the 60-day delayed El program had similar effects compared with the El implemented at the beginning. 
A third group retrospectively recruited was taken into the analyses as a reference group. At the endpoint of T2, all outcome measures were compared among the EI-SC, SC-El, and reference groups using the oneway ANOVA and the Bonferroni test as a post hoc multiple comparisons.

\section{Results}

A total of 138 infants were eligible for the RCT trial; 65 infants were excluded, resulting in 73 objects being randomly allocated, 37 in the El-SC group, and 36 in the SC-El group. Besides, 33 infants were retrospectively recruited as the reference group (Fig. 1). No difference was noted among the three groups regarding demographic characteristics. The baseline TIMP, DQ, and anthropometric measures between the EI-SC group and SC-El group were also comparable (Table 1). 
Table 1

Baseline characteristics and assessment (TO)

\begin{tabular}{|c|c|c|c|c|c|}
\hline & $\begin{array}{l}\text { El-SC group } \\
(n=37)\end{array}$ & $\begin{array}{l}\text { SC-El group } \\
(n=36)\end{array}$ & $\begin{array}{l}\text { Reference } \\
\text { group } \\
(n=33)\end{array}$ & $\begin{array}{l}\text { For } \\
X 2\end{array}$ & $\mathbf{p}$ \\
\hline Gestational age (weeks) & $30.03 \pm 1.79$ & $30.72 \pm 1.45$ & $30.06 \pm 1.39$ & 2.264 & 0.109 \\
\hline Birth weight (gram) & $\begin{array}{l}1432.14 \pm \\
200.00\end{array}$ & $\begin{array}{l}1509.28 \pm \\
201.56\end{array}$ & $\begin{array}{l}1511.18 \pm \\
187.06\end{array}$ & 1.899 & 0.155 \\
\hline $\begin{array}{l}\text { Corrected gestational age } \\
\text { (weeks) }\end{array}$ & $36.92 \pm 0.83$ & $36.64 \pm 0.90$ & $36.97 \pm 1.19$ & 1.178 & 0.312 \\
\hline Weight at discharge (gram) & $\begin{array}{l}2529.19 \pm \\
360.30\end{array}$ & $\begin{array}{l}2453.06 \pm \\
271.02\end{array}$ & $\begin{array}{l}2504.94 \pm \\
340.54\end{array}$ & 0.776 & 0.463 \\
\hline Female/male & $25 / 12$ & $20 / 16$ & $19 / 14$ & 1.258 & 0.053 \\
\hline $\begin{array}{l}\text { Duration of maternal education } \\
\text { (years) }\end{array}$ & $14.24 \pm 2.19$ & $14.58 \pm 2.82$ & $14.82 \pm 3.20$ & 0.390 & 0.678 \\
\hline TIMP score & $52.76 \pm 9.19$ & $50.81 \pm 8.24$ & N/A & 0.911 & 0.343 \\
\hline DQ total score & $83.43 \pm 6.61$ & $80.28 \pm 9.99$ & N/A & 2.543 & 0.115 \\
\hline Gross motor & $82.78 \pm 14.46$ & $79.47 \pm 16.33$ & N/A & 0.843 & 0.362 \\
\hline Fine motor & $86.81 \pm 27.71$ & $78.28 \pm 26.08$ & N/A & 1.834 & 0.180 \\
\hline Adaptiveness & $84.16 \pm 19.94$ & $80.56 \pm 30.02$ & N/A & 0.367 & 0.546 \\
\hline Social & $79.35 \pm 20.91$ & $81.94 \pm 18.59$ & N/A & 0.313 & 0.578 \\
\hline Language & $84.19 \pm 14.16$ & $81.17 \pm 21.25$ & N/A & 0.514 & 0.476 \\
\hline \multicolumn{6}{|l|}{ Physical growth } \\
\hline Length (cm) & $47.45 \pm 2.17$ & $46.74 \pm 1.82$ & $47.04 \pm 1.95$ & 1.182 & 0.311 \\
\hline Weight (kg) & $2.89 \pm 0.81$ & $2.78 \pm 0.72$ & $2.80 \pm 0.65$ & 0.225 & 0.799 \\
\hline Head circumference $(\mathrm{cm})$ & $32.07 \pm 1.27$ & $31.98 \pm 1.57$ & $32.21 \pm 1.70$ & 0.204 & 0.816 \\
\hline
\end{tabular}

\section{Delta Change (t1-t0)}

After a 30-day El, the El-SC group had more significant delta change than SC-El group in TIMP, and physical growth (weight, length, and $\mathrm{HC})(\mathrm{p}<0.05)$. However, no difference in general and individual subdomain DQ was noted between groups $(p>0.05)$ (Table 2). 
Table 2

Increment of neurodevelopment score and physical growth between T1 and TO

\begin{tabular}{|lllll|}
\hline & $\begin{array}{l}\text { El-SC group } \\
(\mathbf{n}=\mathbf{3 4})\end{array}$ & $\begin{array}{l}\text { SC-El group } \\
(\mathbf{n}=\mathbf{3 1})\end{array}$ & $\boldsymbol{F}$ & $\mathbf{p}$ \\
\hline TIMP score & $30.12 \pm 8.85$ & $25.35 \pm 8.4$ & 5.059 & 0.028 \\
\hline DQ total score & $4.08 \pm 1.34$ & $3.77 \pm 1.03$ & 1.023 & 0.316 \\
\hline Gross motor & $5.29 \pm 2.20$ & $4.42 \pm 2.87$ & 1.923 & 0.170 \\
\hline Fine motor & $1.88 \pm 0.81$ & $2.13 \pm 1.73$ & 0.560 & 0.457 \\
\hline Adaptiveness & $3.62 \pm 2.40$ & $2.71 \pm 1.85$ & 2.880 & 0.095 \\
\hline Social & $4.29 \pm 3.25$ & $5.45 \pm 3.73$ & 1.785 & 0.186 \\
\hline Language & $5.29 \pm 3.85$ & $4.16 \pm 1.92$ & 2.180 & 0.145 \\
\hline Physical growth & & & & \\
\hline Length (cm) & $7.07 \pm 1.93$ & $6.01 \pm 1.84$ & 5.069 & 0.028 \\
\hline Weight (kg) & $1.49 \pm 0.63$ & $1.21 \pm 0.46$ & 4.095 & 0.047 \\
\hline Head circumference $(\mathrm{cm})$ & $3.92 \pm 1.10$ & $3.31 \pm 1.16$ & 4.705 & 0.034 \\
\hline
\end{tabular}

\section{Delta Change (t2-t0)}

In terms of delta change from T2 to T0, there was no significant difference in all outcomes between the El-SC group and SC-El group ( $p>0.05)$. However, the marginal difference was noted regarding the language development and weight increment, with a p-value of 0.085 , and 0.065 , respectively (Table 3 ). 
Table 3

Increment of neurodevelopment score and physical growth between T2 and T0

\begin{tabular}{|lllll|}
\hline & $\begin{array}{l}\text { El-SC group } \\
(\mathbf{n}=\mathbf{2 8})\end{array}$ & $\begin{array}{l}\text { SC-El group } \\
(\mathbf{n}=\mathbf{2 9})\end{array}$ & $\boldsymbol{F}$ & $\mathbf{p}$ \\
\hline TIMP score & $60.96 \pm 9.92$ & $58.10 \pm 8.98$ & 1.304 & 0.258 \\
\hline DQ total score & $8.26 \pm 1.05$ & $7.92 \pm 1.84 \mathrm{~s}$ & 0.725 & 0.398 \\
\hline Gross motor & $11.39 \pm 4.48$ & $9.52 \pm 4.99$ & 2.227 & 0.141 \\
\hline Fine motor & $5.14 \pm 2.17$ & $5.83 \pm 2.89$ & 1.016 & 0.318 \\
\hline Adaptiveness & $7.07 \pm 3.69$ & $7.10 \pm 3.60$ & 0.001 & 0.974 \\
\hline Social & $8.82 \pm 5.14$ & $10.28 \pm 6.41$ & 0.890 & 0.350 \\
\hline Language & $8.86 \pm 4.93$ & $6.86 \pm 3.55$ & 3.085 & 0.085 \\
\hline Physical growth & & & & \\
\hline Length (cm) & $12.68 \pm 1.93$ & $12.00 \pm 1.87$ & 1.807 & 0.184 \\
\hline Weight (kg) & $2.80 \pm 0.51$ & $2.54 \pm 0.52$ & 3.533 & 0.065 \\
\hline Head circumference $(\mathrm{cm})$ & $7.99 \pm 1.21$ & $7.55 \pm 1.30$ & 1.689 & 0.199 \\
\hline
\end{tabular}

\section{Assessment At The Endpoint Of T2}

At the endpoint of T2, the EI-SC and SC-El group had similar TIMP, and anthropometric measures, which were significantly higher than that of the reference group by the Bonferroni test. In terms of general and subdomain DQ, no difference was noted among the three groups $(p>0.05)$ (Table 4). 
Table 4

Comparison of the outcomes at T2 assessment

\begin{tabular}{|c|c|c|c|c|c|}
\hline & $\begin{array}{l}\text { El-SC group } \\
(n=28)\end{array}$ & $\begin{array}{l}\text { SC-El group } \\
(n=29)\end{array}$ & $\begin{array}{l}\text { Reference } \\
\text { group } \\
(n=33)\end{array}$ & For $\chi 2$ & $p$ \\
\hline TIMP score & $\begin{array}{l}113.54 \pm \\
12.05^{\mathrm{a}}\end{array}$ & $\begin{array}{l}109.33 \pm \\
11.005^{a}\end{array}$ & $101.18 \pm 12.98^{b}$ & 8.332 & $\varangle 0.001$ \\
\hline DQ total score & $90.56 \pm 6.14$ & $87.08 \pm 10.34$ & $85.06 \pm 7.14$ & 1.538 & 0.130 \\
\hline Gross motor & $94.50 \pm 16.50$ & $89.00 \pm 17.71$ & $84.94 \pm 14.16$ & 2.675 & 0.075 \\
\hline Fine motor & $90.36 \pm 21.88$ & $83.10 \pm 21.54$ & $81.55 \pm 15.63$ & 1.673 & 0.194 \\
\hline Adaptiveness & $89.04 \pm 19.13$ & $90.55 \pm 30.54$ & $88.45 \pm 21.00$ & 0.062 & 0.940 \\
\hline Social & $88.57 \pm 22.56$ & $88.31 \pm 18.73$ & $85.70 \pm 25.86$ & 0.153 & 0.858 \\
\hline Language & $90.57 \pm 16.62$ & $84.66 \pm 13.74$ & $84.67 \pm 10.50$ & 1.772 & 0.176 \\
\hline \multicolumn{6}{|l|}{ Physical growth } \\
\hline Length (cm) & $59.83 \pm 2.77^{a}$ & $58.61 \pm 2.61^{\mathrm{a}}$ & $56.53 \pm 2.45^{\mathrm{b}}$ & 12.614 & $\nabla 0.001$ \\
\hline Weight (kg) & $5.56 \pm 0.92^{\mathrm{a}}$ & $5.24 \pm 0.95^{\mathrm{a}}$ & $4.74 \pm 0.99^{b}$ & 5.547 & 0.005 \\
\hline $\begin{array}{l}\text { Head circumference } \\
(\mathrm{cm})\end{array}$ & $38.91 \pm 2.15^{a}$ & $39.38 \pm 1.93^{a}$ & $38.20 \pm 2.15^{\mathrm{b}}$ & 5.492 & 0.006 \\
\hline
\end{tabular}

\section{Discussions}

El means the intervention should be carried out as soon as possible, commonly started within the first 12 months of life. Post-discharge El has been widely performed in the follow-up clinics or at a communitybased setting, which imposes high costs for the health care system. Taking Massachusetts, United States, as an example, the mean cost per infant for El was 6614 dollars among the infants born at $24 \otimes 31$ weeks' gestation, with a total El cost of 65910379 dollars for all infants between 2009 and 2012(14). Such a huge number implied that the El program might not be affordable in all countries, even in some developed countries. Meanwhile, some factors, such as traffic barriers, household overwork, and expensive accommodation cost, also limit the parents to access the El programs. Therefore, exploring a low-cost, family-based, flexible El program was urgent for the medical providers and some families in need.

Amid this background, Sgandurra et al. developed a new technological smart modular system as a telerehabilitation tool for El, which could be implemented by parents at home under the orientation of rehabilitation staff. They demonstrated that this home-based El significantly improved infant motor and 
visual development(15). However, drawbacks also was evident; the occurrence of technique errors seemed unavoidable for an internet-based system, and getting acquainted with a new system was also a challenge for some parents (16). In this study, we conducted an innovative approach to carry out the home-based El program, which was independent of any technological equipment, and was free of knowledge gap for parents to perform it. Our study demonstrated that this El program could promote motor development of EPI, as well as physical growth.

TIMP is the primary outcome measure in this study. Our results of RCT period (T1-T0) showed the delta change of TIMP in El-SC group was significantly higher than in SC-El group, which was consistent with Sgandurra's study(15); however, it conflicted to many previous studies, which did not show any significant effect of El on motor development $(4,6,17)$. Some factors might contribute to the discrepancy. The frequency of El performed in this study is once daily over four weeks, much higher than other studies which mostly ranged from once weekly to twice weekly(4). Sgandurra et al. demonstrated that amout of El performance could affect the improvement of motor development (15). Meanwhile, our program added some good evidence-based interventions to the basic intervention, including body massage and kangaroo care. Body massage has been shown to effectively enhance motor development in various children population, including infants $(18,19)$. The effect of kangaroo care on infant development was also well confirmed(20). Massage and kangaroo care superimposed on basic intervention might generate a synergistic effect. Besides, distinct assessment tools might also lead to the different results. Most previous studies used the Bayley physical development index (BPDI) to assess motor development, whereas our study used the TIMP instead. TIMP was approved to be a reliable and valid measurement of motor development, showing a highly significant correlation with BPDI(21); TIMP is suitable for assessing infants $<6$ months, while six months is the lower age limit for BPDI. Some studies indicated that the effect of El on motor development was significant in infancy, but was not noted in the long term. Therefore, a positive result in this study might be partially due to a relatively earlier assessment.

RCT did not show any significant difference in the DQ score between El-SC group and SC-El group. Improvement of motor development demonstrated by TIMP was not present during DQ assessment, which may be attributed to the structural limitation of the DQ tool. Each subdomain of DQ only has one or two items under each month, scored as "observed" or "unobserved"; some minor changes cannot be specified. Meanwhile, DQ might delay identifying some subtle improvement, even some of which have already been noted by the parents(22). In terms of physical growth, this El program generally promoted the growth of weight, length, and $\mathrm{HC}$, which was considered the results of a combination of many factors. Body massage has been shown to have the potential to improve gastrointestinal function, and ultimately increase physical growth(23). Literature also indicated that Kangaroo care could shape preterm infant sleep behavior and prolong the duration of deep sleep(24); a longer duration of deep sleep is a good predictor of optimal physical growth(25). The integrated El program also could improve infant feeding behavior, increase feeding volume, and positively affect physical growth $(26,27)$.

We conducted the open phase after the RCT, creditable from the consideration of ethics. No difference was noted between the EI-SC and SC-El group regarding delta change (T2-T0) in all outcomes, which gave 
rise to the inference that the 60-day delayed El had a similar effect with the El at the beginning. Assessment at T2 endpoint also confirmed that no difference in TIMP and physical growth measures existed between the EI-SC and SC-El group, but both were much higher than the reference group. It was an encouraging result for some parents who are concerned about the effect of the delayed intervention for their infants due to a variety of causes. However, currently, to carry out an El program as soon as possible is still recommended.

Our study had certain limitations. We did not perform a long-term follow-up, hampering the evaluation of the long-term effect of this El program. Meanwhile, the parent's performance should alert the effectiveness of the El program. It was impossible to keep all parent's performance identical in this study. Fortunately, during the teaching session, our researchers should ensure that every parent had the basic skills to perform El. In this study, we also cannot completely rule out the situation that some parents performed additional El than the study protocol; even the parents have initially signed the consent to comply with the study protocol.

\section{Conclusions}

This study introduced a home-based, post-discharge El program, which was less-cost, particularly suitable in medical source-limited regions. This El program could significantly promote motor development and physical growth of EPI, at least in a short-term period. However, there is still a need to perform long-term follow-up to identify the long-term effect of this program on infant development.

\section{Abbreviations}

EPI: early preterm infants; El: early intervention; RCT: randomized controlled trial; El-SC group: first early intervention, then standard care group; SC-El: first standard care, then early intervention group. HC: head circumference; TIMP: test of infant motor performance; DQ: development quotient; BPDI: Bayley physical development index

\section{Declarations}

\section{Ethics approval and consent to participate}

Subjects were enrolled in the study after written informed consent obtained from their parents. This study was approved by the Institutional Review Board of Children's Hospital of Chongqing Medical University (No.2019-216).

\section{Consent for publication}

A written consent for publication has obtained from the parents of the infant shown in our supplementary video. 
Availability of data and materials

The datasets used and/or analyzed during the current study are available from the corresponding author on reasonable request.

\section{Competing interests}

The authors declare that they have no competing interests.

\section{Funding}

This work was supported by the Chongqing Science and Technology Commission (No.cstc2018jscxmsybX0071). The funder did not play a role in any aspect of the study, including design, data collection, analysis or manuscript writing.

\section{Authors' contributions}

JF and JHW contributed to study conceptualization, data acquisition and data analysis, drafting the initial manuscript, and revising the manuscript before submission. JHW and XG contributed to the data acquisition, and writing the initial manuscript. XHZ, RYH and SSH contributed to data analysis, and revising the manuscript. MY, YJS and XJT contributed to data analysis, compiling tables and figures. LJH contributed to study conceptualization and design, data analysis, reviewing and revising the manuscript. All authors made substantial contributions to the conduct of the study and approved the final manuscript as submitted.

\section{Acknowledgements}

Not applicable

\section{References}

1. Hafstrom M, Kallen K, Serenius F, Marsal K, Rehn E, Drake H, et al. Cerebral Palsy in Extremely Preterm Infants. Pediatrics. 2018;141:e20171433.

2. Vohr BR, Wright LL, Poole WK, McDonald SA. Neurodevelopmental outcomes of extremely low birth weight infants <32 weeks' gestation between 1993 and 1998. Pediatrics. 2005;116:635-43.

3. Spittle A, Orton J, Anderson P, Boyd R, Doyle LW. Early developmental intervention programmes posthospital discharge to prevent motor and cognitive impairments in preterm infants. The Cochrane database of systematic reviews. 2012;12:Cd005495.

4. Spittle A, Orton J, Anderson PJ, Boyd R, Doyle LW. Early developmental intervention programmes provided post hospital discharge to prevent motor and cognitive impairment in preterm infants. The Cochrane database of systematic reviews. 2015:Cd005495. 
5. Litt JS, Glymour MM, Hauser-Cram P, Hehir T, McCormick MC. Early Intervention Services Improve School-age Functional Outcome Among Neonatal Intensive Care Unit Graduates. Acad Pediatr. 2018;18:468-74.

6. Wu YC, Leng $\mathrm{CH}$, Hsieh WS, Hsu CH, Chen WJ, Gau SS, et al. A randomized controlled trial of clinicbased and home-based interventions in comparison with usual care for preterm infants: effects and mediators. Res Dev Disabil. 2014;35:2384-93.

7. Rocha-Ferreira E, Hristova M. Plasticity in the neonatal brain following hypoxic-ischaemic injury. Neural Plast. 2016;2016:4901014.

8. Borghini A, Habersaat S, Forcada-Guex M, Nessi J, Pierrehumbert B, Ansermet F, et al. Effects of an early intervention on maternal post-traumatic stress symptoms and the quality of mother-infant interaction: the case of preterm birth. Infant Behav Dev. 2014;37:624-31.

9. Jackson YM. The relationship between early intervention service dosage and child outcome growth patterns: Middle Tennessee State University; 2017.

10. Alderman H, Behrman JR, Glewwe P, Fernald L, Walker S. Evidence of impact of interventions on growth and development during early and middle childhood. Disease Control Priorities, (Volume 8): Child and Adolescent Health and Development. 2017:1790.

11. Cioni G, Inguaggiato E, Sgandurra G. Early intervention in neurodevelopmental disorders: underlying neural mechanisms. Dev Med Child Neurol. 2016;58:61-6.

12. Chiquetti EMdS, Valentini NC, Saccani R. Validation and Reliability of the Test of Infant Motor Performance for Brazilian Infants. Phys Occup Ther Pediatr. 2020:1-16.

13. Matsushima S, Ozawa K, Sugibayashi R, Ogawa K, Tsukamoto K, Miyazaki O, et al. Neurodevelopmental impairment at 3 years of age after fetoscopic laser surgery for twin-to-twin transfusion syndrome. Prenat Diagn. 2020:1013-9.

14. Clements KM, Barfield WD, Ayadi MF, Wilber N. Preterm birth-associated cost of early intervention services: an analysis by gestational age. Pediatrics. 2007;119:e866-74.

15. Sgandurra G, Lorentzen J, Inguaggiato E, Bartalena L, Beani E, Cecchi F, et al. A randomized clinical trial in preterm infants on the effects of a home-based early intervention with the 'CareToy System'. PLoS One. 2017;12:e0173521.

16. Sgandurra G, Bartalena L, Cecchi F, Cioni G, Giampietri M, Greisen G, et al. A pilot study on early home-based intervention through an intelligent baby gym (CareToy) in preterm infants. Res Dev Disabil. 2016;53-54:32-42.

17. Dusing SC, Brown SE, Van Drew CM, Thacker LR, Hendricks-Muñoz KD. Supporting Play Exploration and Early Development Intervention From NICU to Home: A Feasibility Study. Pediatr Phys Ther. 2015;27:267-74.

18. Lu WP, Tsai WH, Lin LY, Hong RB, Hwang YS. The Beneficial Effects of Massage on Motor Development and Sensory Processing in Young Children with Developmental Delay: A Randomized Control Trial Study. Dev Neurorehabil. 2019;22:487-95. 
19. Trivedi D. Cochrane Review Summary: Massage for promoting mental and physical health in typically developing infants under the age of six months. Prim Health Care Res Dev. 2015;16:3-4.

20. Moore ER, Bergman N, Anderson GC, Medley N. Early skin-to-skin contact for mothers and their healthy newborn infants. Cochrane database of systematic Reviews. 2016;5:CD003519.

21. Kim SA, Lee YJ, Lee YG. Predictive Value of Test of Infant Motor Performance for Infants based on Correlation between TIMP and Bayley Scales of Infant Development. Ann Rehabil Med. 2011;35:8606.

22. Matsuishi T, Ishibashi S, Kamiya Y, Shoji J, Yamashita Y, Fukuda S, et al. Early intervention for verylow-birth-weight infants. Brain Dev. 1998;20:18-21.

23. Choi H, Kim S-J, Oh J, Lee M-N, Kim S, Kang K-A. The effects of massage therapy on physical growth and gastrointestinal function in premature infants: A pilot study. Journal of Child Health Care. 2016;20:394-404.

24. Bastani F, Rajai N, Farsi Z, Als H. The effects of kangaroo care on the sleep and wake states of preterm infants. J Nurs Res. 2017;25:231-9.

25. Tham EK, Schneider N, Broekman BF. Infant sleep and its relation with cognition and growth: a narrative review. Nature and science of sleep. 2017;9:135.

26. Fontana C, Menis C, Pesenti N, Passera S, Liotto N, Mosca F, et al. Effects of early intervention on feeding behavior in preterm infants: A randomized controlled trial. Early Hum Dev. 2018;121:15-20.

27. Ferreira Hda S, Xavier Júnior AF, de Assunção ML, Dos Santos EA, Horta BL. Effect of breastfeeding on head circumference of children from impoverished communities. Breastfeed Med. 2013;8:294301.

\section{Figures}




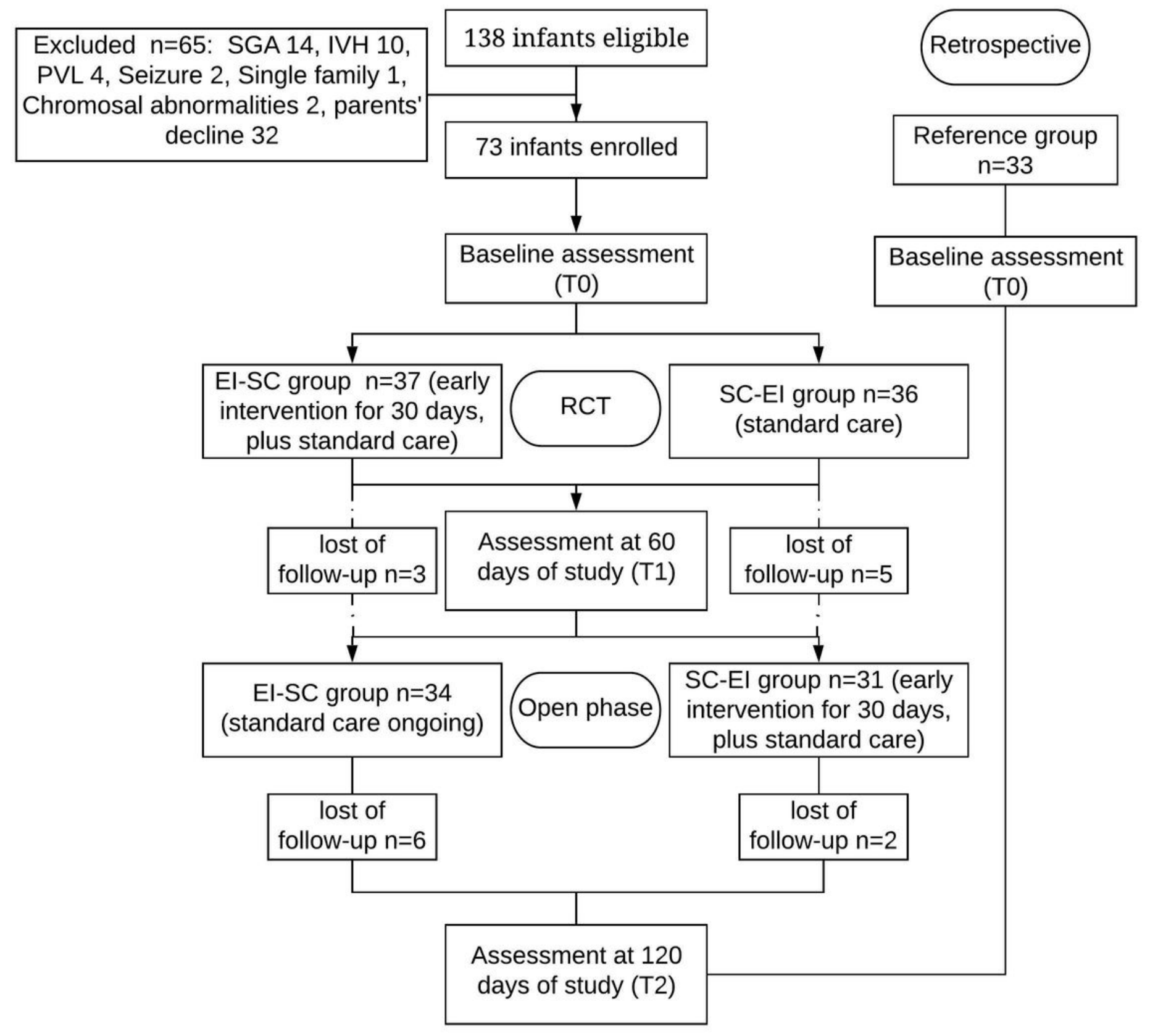

Figure 1

Study flow diagram. SGA, small for gestational age; IVH, intraventricular hemorrhage; PVL, periventricular leukomalacia; RCT, randomized controlled trials;

\section{Supplementary Files}

This is a list of supplementary files associated with this preprint. Click to download.

- CONSORT2010Checklist.doc

- Additionalfile1.docx 